Disclosure of Interest: None declared

DOI: 10.1136/annrheumdis-2017-eular.2709

\section{FRI0581 HEPATITIS B INCREASE MORTALITY IN PATEINTS WITH OSTEOPOROTIC VERTEBRAL FRACTURES}

Y.-C. Chen, C.-H. Ko. Rheumatology, Kaohsiung Chang Gung Memorial Hospital, Kaohsiung, Taiwan, Province of China

Background: Hepatitis B virus (HBV) is a major cause of chronic liver diseases worldwide, particularly cirrhosis and hepatocellular carcinoma, and the most important liver disease in Taiwan. However, little is known the impact of hepatitis b on osteoporotic patients.

Objectives: This study aimed to determine if hepatitis $b$ can increase the risk of mortality in patients with osteoporotic vertebral fracture.

Methods: This retrospective study reviewed of cases of osteoporosis patients with acute vertebral fractures between 2001 and 2008. All associated co-morbidities were recorded. Kaplan-Meier and cox regression analysis were performed.

Results: There were 432 patients with acute vertebral fractures. The mean age of $72.85 \pm 9.28$. $31(7.2 \%)$ patients had chronic hepatitis b. Using the Kaplan-Meier curve, hepatitis $b$ had a significant effect on mortality $(p<0.001$, by log rank text). After adjusting for potential confounders, patients with hepatitis $b$ still had a high mortality rate ( $p=0.019$; HR: $2.436 \sim 5.136) 2.137,95 \% \mathrm{Cl}: 1.156 \sim 5.136)$. The mortality rate also increased among smokers ( $p=0.026$; HR: $3.6043 .891 ; 95 \% \mathrm{Cl}$ : 1.056 12.301)

Table 1. Multivariable Cox regression analysis of the hazard ratios for adjacent fracture

\begin{tabular}{lccc}
\hline Variables & Regression coefficient & $\mathrm{P}$ value & $\mathrm{HR}(95 \mathrm{Cl})$ \\
\hline Gender & -0.127 & 0.764 & $0.88(0.383-0.022)$ \\
Age & 0.004 & 0.785 & $1.004(0.976-1.032)$ \\
Smoking & 1.282 & 0.041 & $3.604(1.056-12.301)$ \\
Alcohol consumption & -0.701 & 0.396 & $0.496(0.098-2.508)$ \\
Body mass index $\left(\mathrm{kg} / \mathrm{m}^{2}\right)$ & 0.024 & 0.446 & $1.024(0.964-1.088)$ \\
Hepatitis B & 0.891 & 0.019 & $2.436(1.156-5.136)$ \\
Diabetes & -0.185 & 0.538 & $0.831(0.461-1.498)$ \\
Hypertension & -0.271 & 0.31 & $0.762(0.451-1.288)$ \\
Stroke & -0.696 & 0.248 & $0.499(0.153-1.625)$ \\
Kidney disease & 0.809 & 0.145 & $2.246(0.758-6.656)$ \\
Cardiovascular disease & 0.037 & 0.946 & $1.038(0.351-3.069)$ \\
Pulmonary disease & -0.971 & 0.349 & $0.379(0.050-2.883)$ \\
\hline
\end{tabular}

Abbreviations: HR, hazard ratio; SE, standard error.

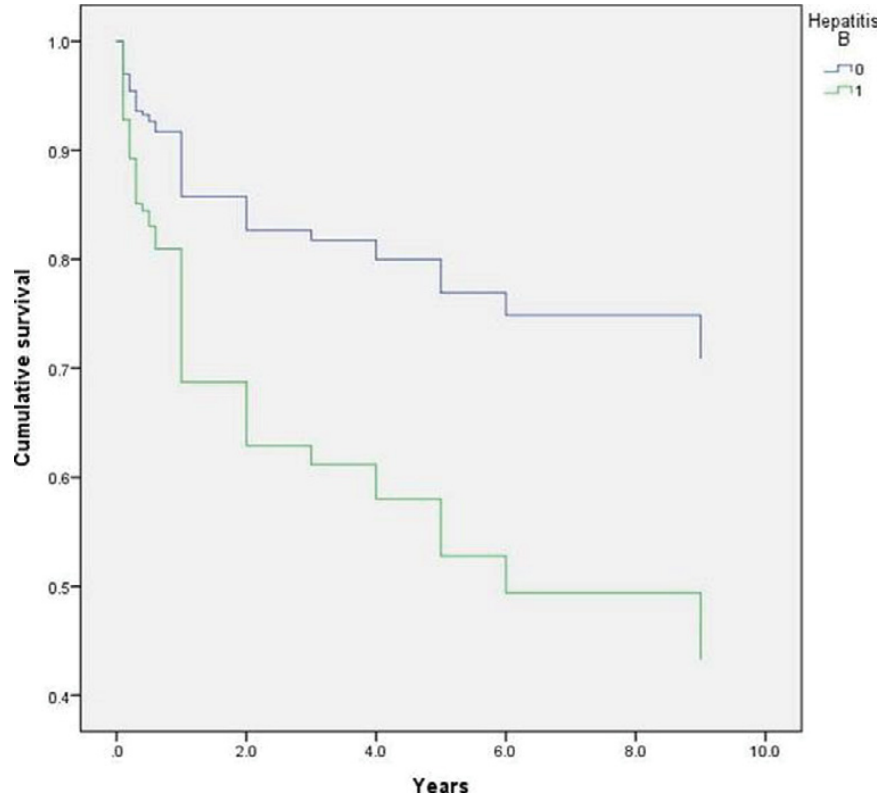

Conclusions: In this study, hepatitis b increase mortality in osteoporotic vertebral fracture patients. So we should pay attention to this group in osteoporotic management.

References:

[1] Chong-Shan Wang et al. Am. J. Trop. Med. Hyg., 66(4), 2002, pp. 389-393. Acknowledgements: We thank Kaohsiung Chang Gang Memorial Hospital for data support.

Disclosure of Interest: None declared

DOI: 10.1136/annrheumdis-2017-eular.3614

\section{FRIDAY, 16 JUNE 2017 Other orphan diseases}

\section{FRI0582 OPEN-LABEL, MULTICENTER, DOSE-ESCALATING PHASE II CLINICAL TRIAL ON THE SAFETY AND EFFICACY OF TADEKINIG ALPHA IN ADULT ONSET STILL'S DISEASE}

C. Gabay ${ }^{1}$, B. Fautrel ${ }^{2}$, J. Rech ${ }^{3}$, F. Spertini ${ }^{4}$, E. Feist ${ }^{5}$, I. Koetter ${ }^{6}$ E. Hachulla ${ }^{7}$, J. Morel ${ }^{8}$, T. Schaeverbeke ${ }^{9}$, M.A. Hamidou ${ }^{10}$, T. Martin ${ }^{11}$, B. Hellmich ${ }^{12}$, P. Lamprecht ${ }^{13}$, H. Schultze-Koops ${ }^{14}$, A. Sleight ${ }^{15}$, E. Schiffrin ${ }^{15}$ ${ }^{1}$ Rheumatology, University Hospitals of Geneva, Geneva, Switzerland;

${ }^{2}$ Rheumatology, Pitié Salpetrière University Hospital, Paris, France; ${ }^{3}$ Department of Medicine 3, Rheumatology and Immunology, Universitätsklinikum Erlangen, Erlangen, Germany: ${ }^{4}$ Division of Immunology and Allergy, CHUV, Lausanne, Switzerland; ${ }^{5}$ Rheumatology, Charité Universitatsmedizin, Berlin; ${ }^{6}$ Asklepios Klinikum, Hamburg, Germany; ${ }^{7} \mathrm{CHRU}$ de Lille - Hopital Claude Huriez, Lille; ${ }^{8}$ Department of Rheumatology, University and CHU of Montpellier, Montpellier;

${ }^{9}$ Rheumatology, Hopital Pellegrin, Bordeaux; ${ }^{10} \mathrm{CHU}$ de Nantes, Nantes:

${ }^{11}$ University Hospital of Strasbourg, Strasbourg. France; ${ }^{12}$ Klinik fur Innere Medizin, Rheumatologie und immunologie, Medius Klinik, Kirchheim unter Teck

${ }^{13}$ Rheumatology, University of Lübeck, Lübeck; ${ }^{14}$ Medizinische Klinik und

Poliklinik IV, LMU, Muenchen, Germany; ${ }^{15}$ Ab2 Bio, Lausanne, Switzerland

Background: Adult Onset Still's Disease (AOSD) is a severe systemic autoinflammatory disease. Elevated interleukin (IL)-18 levels correlate with AOSD disease activity, suggesting a central role

Objectives: To examine the safety and efficacy of IL-18 blockade in AOSD with the administration of Tadekinig alpha, a recombinant human IL-18 binding protein Methods: Patients (Pts) with AOSD according to Yamaguchi criteria were included if they had been previously treated for at least one month with corticosteroids (CS). The pts received either $80 \mathrm{mg}$ (group 1) or $160 \mathrm{mg}$ (group 2) Tadekinig alpha sc three times weekly for 12 weeks. Oral CS and/or conventional DMARDs were allowed at stable dose in combination with Tadekinig alpha. After 3 wks, group 1 pts qualified as non responders (CRP levels did not decrease by $\geq 50 \%$ from baseline values or absence of temperature normalization) could be up-dosed to $160 \mathrm{mg}$ for an additional 12 weeks. The primary endpoint was safety and secondary endpoints included early signs of efficacy

Results: 23 pts were included (10 and 13 in group 1 and group 2, respectively). 6 pts of group 1 were up-dosed to $160 \mathrm{mg}$. Baseline characteristics and safety and efficacy results are described in Table 1. Most adverse events (AEs) (47) were considered as mild or moderate. Three serious AEs (SAE) were reported including two that were considered not related to the study drug and one possibly related according to the investigator (toxic optic neuropathy). Four premature discontinuations were related to $\mathrm{AEs}$, including 3 cases of injection site reactions and 1 SAE. Systemic response as defined by $\geq 70 \%$ decrease of serum C-reactive protein (CRP) or normalization of CRP and ferritin levels was obtained in 2/10 and 6/13 pts of groups 1 and 2, respectively in the ITT analysis, and in 2/9 and $6 / 9$ pts of groups 1 and 2, respectively, in the PP analysis.

Table 1. Baseline characteristics and safety and efficacy results

\begin{tabular}{lcc}
\hline & $\begin{array}{c}\text { Group 1 }(80 \mathrm{mg}) \\
(\mathrm{N}=10)\end{array}$ & $\begin{array}{c}\text { Group 2 (160 mg) } \\
(\mathrm{N}=13)\end{array}$ \\
\hline Age, median (IQR) & $49.5(34.3-58.8)$ & $35(30-58)$ \\
Median disease duration, months (IQR) & $25.5(8.7-44.2)$ & $11.6(2.1-37.6)$ \\
Pts with previous conventional DMARDs & 6 & 7 \\
Pts with previous biological DMARDs & 4 & 5 \\
Pts with SAEs & 2 & 1 \\
Discontinuations due to AEs & 1 & 3 \\
Discontinuations due to lack of response & 1 & 2 \\
Arthritis improvement $(\geq 20 \%$ reduction in tender & & \\
$\quad$ and swollen joint count) & & $4 / 10$ \\
Skin rash resolution & & $2 / 5$ \\
CRP level reduction by $\geq 70 \% * *$ & $6 / 7$ & $6 / 13$ \\
Normalization of ferritin levels & & $1 / 5$ \\
\hline
\end{tabular}

*Number of Pts, ${ }^{*}$ Denominator corresponds to Pts with clinical manifestations or abnormal values at baseline.

Conclusions: IL-18 blockade by Tadekinig alpha has an acceptable safety profile and is efficacious, in particular at the $160 \mathrm{mg}$ dosage, in patients with refractory AOSD

Disclosure of Interest: C. Gabay Grant/research support from: AB2 Bio, Roche, Pfizer, Consultant for: AB2 Bio, Roche, Pfizer, MSD, BMS, AbbVie, Sanofi, B. Fautrel: None declared, J. Rech: None declared, F. Spertini: None declared, E. Feist: None declared, I. Koetter: None declared, E. Hachulla: None declared, J. Morel: None declared, T. Schaeverbeke: None declared, M. Hamidou: None declared, T. Martin: None declared, B. Hellmich: None declared, P. Lamprecht: None declared, H. Schultze-Koops: None declared, A. Sleight Employee of: Ab2 Bio, E. Schiffrin Employee of: Ab2 Bio

DOI: 10.1136/annrheumdis-2017-eular.2336 